\title{
Excited state kinetics of anthracene-bridge-aniline intramolecular exciplexes
}

Thyrhaug, Erling; Hammershøj, Peter; Kjær, Kasper Skov; Sørensen, Thomas Just; Harrit, Niels H.; Nielsen, Martin Meedom; Bechgaard, Klaus

Published in:

Photochemical \& Photobiological Sciences

Link to article, DOI:

10.1039/c4pp00073k

Publication date:

2014

Document Version

Publisher's PDF, also known as Version of record

Link back to DTU Orbit

Citation (APA):

Thyrhaug, E., Hammershøj, P., Kjær, K. S., Sørensen, T. J., Harrit, N. H., Nielsen, M. M., \& Bechgaard, K. (2014). Excited state kinetics of anthracene-bridge-aniline intramolecular exciplexes. Photochemical \& Photobiological Sciences, 13(7), 1093-1105. https://doi.org/10.1039/c4pp00073k

\section{General rights}

Copyright and moral rights for the publications made accessible in the public portal are retained by the authors and/or other copyright owners and it is a condition of accessing publications that users recognise and abide by the legal requirements associated with these rights.

- Users may download and print one copy of any publication from the public portal for the purpose of private study or research.

- You may not further distribute the material or use it for any profit-making activity or commercial gain

- You may freely distribute the URL identifying the publication in the public portal 


\title{
Photochemical \& Photobiological Sciences
}

\section{PAPER}

Cite this: Photochem. Photobiol. Sci., 2014, 13, 1093

\section{Excited state kinetics of anthracene-bridge-aniline intramolecular exciplexes $\uparrow$}

\author{
Erling Thyrhaug, ${ }^{a}$ Peter Hammershøj, ${ }^{\star a, b}$ Kasper S. Kjær, ${ }^{\mathrm{b}, \mathrm{c}}$ Thomas Just Sørensen, ${ }^{\mathrm{a}}$ \\ Niels H. Harrit, ${ }^{a, b}$ Martin M. Nielsen ${ }^{c}$ and Klaus Bechgaard ${ }^{a, b}$
}

\begin{abstract}
We report on the synthesis and characterization of fluorescent halogen substituted anthracene-bridgeaniline (ABA) supermolecules that undergo structural reorganization on photoexcitation to form transient complexes. The syntheses were achieved in high yields on a large scale and the molecular structures were confirmed by single crystal X-ray diffraction. The photophysics of the ABA supermolecules were investigated using steady state and time resolved optical spectroscopy. Despite the presence of heavy atoms the series of ABA molecules have high quantum yields of fluorescence from both a locally excited anthracene state (LE) and an excited state complex (exciplex, EP) in non-polar solvents. The kinetics of the excited state processes were established in decalin from the time-resolved emission, and was shown to be strongly influenced by an electron-transfer state (ET). For quantitative studies of the excited state dynamics, the presence of this state required the development of a numerical three-excited-state kinetic model to replace the commonly used two-excited-state model. The experimental results shows that the reaction rates are strongly influenced both by substituents and solvent, illustrating the importance of including all relevant states in the kinetic modeling. Ultimately it is established that the excited state dynamics can conveniently be followed by optical methods, and the applicability of the system as a model system in time-resolved $\mathrm{X}$-ray scattering experiments is discussed.
\end{abstract}

Received 3rd March 2014

Accepted 24th May 2014

DOI: $10.1039 / \mathrm{c} 4 \mathrm{pp} 00073 \mathrm{k}$

www.rsc.org/pps purpose time-resolved absorption and emission spectroscopies especially have proved essential tools in providing a good experimental basis for understanding the photoinduced changes in electronic structure..$^{5-7,9,12-14,23}$ Often the reaction rate constants are the main parameters of interest, and a typical approach to extracting these from the relatively complex optical data has been to assume proportionality between the excited state populations and emission (or transient absorption) intensity. The time dependence of the emission is then fitted to a set of differential equations describing the assumed excited state population dynamics. A number of studies taking this approach are available in literature, typically involving only two excited states in the model: the initially photoexcited local state LE and the reaction product EP. ${ }^{8,22,24-32}$ This truncation of the excited state manifold to only two states, although often a good approximation, will however fail when additional electronic states are close enough in energy to interfere. In this work we extend the typical treatment with a numerical approach involving three excited states to account for, in this case, the presence of an electron transfer state. While optical methods have provided a wealth of information about electronic transformations during chemical reaction, only during the recent two decades have dramatic advances in time-resolved X-ray scattering techniques made it possible to directly gain structural information during 
chemical processes in-solution. Due to the available intensities of X-ray sources however, the majority of solution studies have so far been performed on strongly scattering heavy metal complexes and small heavy-atom (typically iodine) containing molecules, ${ }^{29,33-38}$ although some very recent studies have provided information on both structural and electronic transformations in iron complexes. ${ }^{39,40}$ As they provide complimentary information, these types of X-ray studies could in combination with optical experiments provide unprecedented insight into chemical reaction dynamics. Organic exciplexes provide in this context a good model system for extending time-resolved X-ray studies into the field of organic chemistry, thus taking advantage of new high-brilliance X-ray sources. A number of issues must still be addressed however. The available X-ray intensities have increased dramatically in later years, but in studies of insolution organic complexes incorporation of semi-heavy nuclei such as bromine in the structure is still necessary in order to provide a sufficient scattering signal. Intuitively, such introduction of heavy atoms in the structure of reactants in (singlet-) excited state reactions would be expected to result in decreased efficiency due to reduction of the excited state lifetimes, ${ }^{20}$ and for exciplex formation this effect has been experimentally observed. ${ }^{41}$ The well-studied exciplexes between anthracene and amines are promising in this regard as anthracenes may show increases in excited state lifetime upon halogen substitution, ${ }^{17}$ thus increasing the reaction efficiency. Another practical problem is that intermolecular exciplex rates of formation are diffusion controlled, which drastically limits the achievable total excited state population at reasonable optical densities. One approach to this issue is to link the reactants with a flexible chain, thereby increasing the reaction rate by creating a high local concentration while still having the option of working at low optical densities. In a number of studies of aromatic hydrocarbons (e.g. pyrene, anthracene or naphthalene) and their exciplexes with amines this flexiblelinker approach has been taken, ${ }^{18,22,24,27,31,42,43}$ the results of which illustrate that both length and rigidity of the linker strongly influence the reaction rates. The rates generally appears to be highest when the linker is a propylene chain, ${ }^{20}$ and thus we prepare the anthracene-propylene bridge-aniline (ABA) molecules shown in Fig. 1, while we investigate the

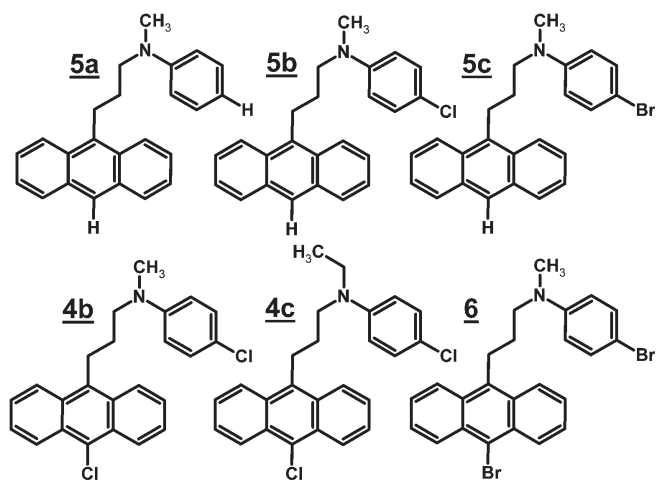

Fig. 1 Schematic structure of anthracene-bridge-aniline (ABA) molecules. effect of bridge substituent size by comparison of $N$-methyl and $N$-ethyl derivatives.

The parent ABA molecule 5a has attracted some attention earlier, ${ }^{21,32,44}$ and its optical properties have been fairly well established. Its emission in low polarity solvents consists of the structured, high energy emission band of a locally excited (LE) anthracene-like state, and a broad emission band at lower energies attributed to the intramolecular exciplex (EP). The emission lifetimes and quantum yields of 5a are quite long and high respectively in low polarity solvents, but, as is typical of exciplexes, both emissive states are dramatically quenched at higher polarity. ${ }^{18,19,23,24,27,31,43}$ As such we study the exciplex formation of the compounds in Fig. 1 most carefully in decalin, with less emphasis on more polar solvents. We conclude that the photophysical properties ABA are now well characterized in low polarity solvents and that these systems could be ideal model systems for time-resolved X-ray studies.

\section{Experimental}

Unless otherwise stated, all starting materials were obtained from commercial suppliers and used as received. Solvents were HPLC grade and were used as received.

\section{Synthesis}

The synthesis of the anthracene-bridge-aniline systems 4-6 followed the route shown in Scheme 1. It is suitable for large scale preparation as it does not require chromatography and all intermediates are crystalline. The synthesis of 3-(9-anthryl)propanoic acid 1 was performed as described by Spivey et al. ${ }^{45}$ 1 was converted to the 3-(9-anthryl)-propanamides 2 a-d via the acid chloride. 1 was treated with oxalyl chloride in dry dichloromethane. After evaporation of the solvent and the surplus of oxalylchloride, the crude acid chlorides were treated with the corresponding $\mathrm{N}$-alkyl aniline and $\mathrm{Et}_{3} \mathrm{~N}$, together with

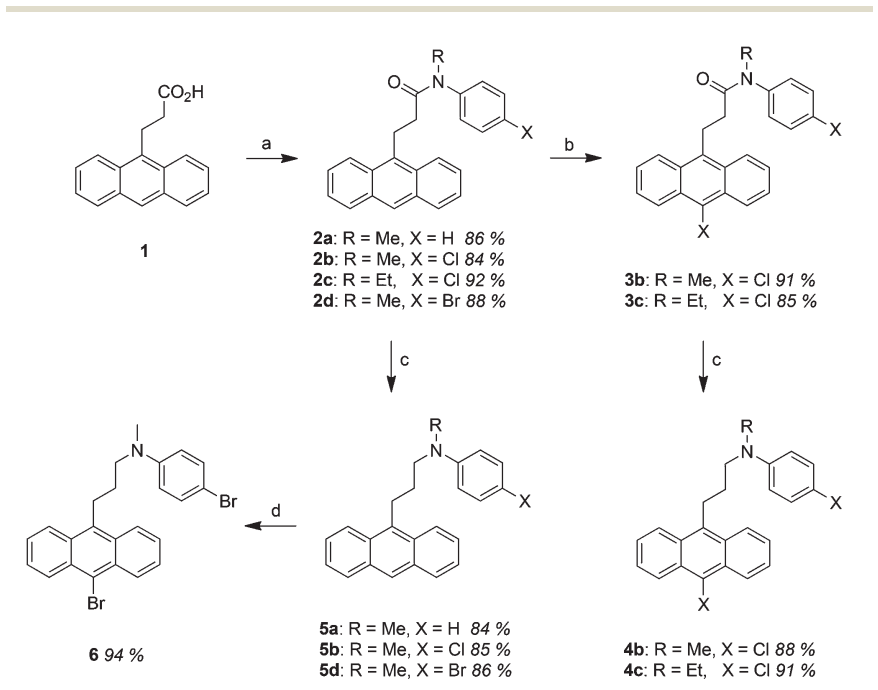

Scheme 1 Synthesis of anthracene-bridge-aniline molecules. (a) $(\mathrm{COCl})_{2}, \mathrm{DMF}, \mathrm{CH}_{2} \mathrm{Cl}_{2}$, aniline, $\mathrm{Et}_{3} \mathrm{~N}$, DMAP, $\mathrm{CH}_{2} \mathrm{Cl}_{2}$. (b) $\mathrm{NCS}, \mathrm{DMF}$. (c) $\mathrm{BH}_{3} \cdot \mathrm{THF}$, dry THF. (d) $\mathrm{Br}_{2}$. 
a catalytic amount of DMAP. The amides were all obtained in good yields. To obtain the dibrominated $N$-(3-(9-anthryl)propyl)- $N$-methylaniline 6, 3-(9-anthryl)- $N$-(phenyl)- $N$-methylpropanamide (2a) was reduced with a solution of $1 \mathrm{M}$ boraneTHF-complex in THF at room temperature to give $\mathrm{N}$-(3(9-anthryl)-propyl)- $N$-methylaniline 5a in $84 \%$ yield. Bromination of 5a with $\mathrm{Br}_{2}$ in $\mathrm{CH}_{2} \mathrm{Cl}_{2}$ gave amine 4-bromo- $N$-(3-(9-(10bromoanthryl))-propyl)- $N$-methylaniline 6 in $94 \%$ yield.

The reduction of the amides 3-(9-anthryl)- $N$-(4-chlorophenyl)- $N$-methyl-propanamide (2b) and 3-(9-anthryl)- $N$-(4-bromophenyl)- $N$-methyl-propanamide (2d) was accomplished using the same procedure as above and yielded $N$-(3-(9-anthryl)propyl)-4-chloro- $N$-methylaniline (5b) and $N$-(3-(9-anthryl)propyl)-4-bromo- $N$-methylaniline (5d) in $85 \%$ and $86 \%$ yields, respectively. Chlorination in the 9-position of anthracene was achieved with equivalent amounts of NCS in DMF in high yields. Borane-THF-complex reduction yielded $N$-(3-(9-(10chloroanthryl))-propyl)-4-chloro- $N$-methylaniline (4b) and $N$-(3(9-(10-chloroanthryl))-propyl)-4-chloro- $N$-ethylaniline $(\mathbf{4 c )}$ in $88 \%$ and $91 \%$ yield, respectively. All structures were characterized by ${ }^{1} \mathrm{H}$ NMR, ${ }^{13} \mathrm{C}$ NMR, GCMS and elemental analysis. Furthermore, the ground state structures of compounds $\mathbf{4 b}, \mathbf{4 c}$, $\mathbf{5 b}, \mathbf{5 d}$, and $\mathbf{6}$ were ascertained by single-crystal X-ray diffraction (Fig. 2 and 3). 9-Chloro-10-methylanthracene (8) and 9-bromo-10-methylanthracene (9) were obtained with small modifications to known procedures. $^{25,46}$ The modifications consisted of improved purification procedures (recrystallization in 5:95 toluene-ethanol) and for 9-chloro-10-methylanthracene (8) an additional aliquot of $\mathrm{CuCl}_{2}$ was added during the reaction from 10-methylanthracene (7). The modifications resulted in higher yields and high purity. Detailed synthetic procedures for all compound can be found in the ESI. $\dagger$

\section{Characterization}

${ }^{1} \mathrm{H}$ NMR and ${ }^{13} \mathrm{C}$ NMR spectra were recorded on a $400 \mathrm{MHz}$ (Varian) instrument (400 MHz for ${ }^{1} \mathrm{H}$ NMR and $100 \mathrm{MHz}$ for ${ }^{13} \mathrm{C} \mathrm{NMR}$ ) or on a $500 \mathrm{MHz}$ (Varian) instrument $(500 \mathrm{MHz}$ for ${ }^{1} \mathrm{H}$ NMR and $125 \mathrm{MHz}$ for ${ }^{13} \mathrm{C}$ NMR). Proton chemical shifts are reported in ppm downfield from tetramethylsilane (TMS) and carbon chemical shifts in ppm downfield of TMS, using the resonance of the solvent as internal standard. Melting points were measured on a Gallenkamp apparatus and are uncorrected. HRMS were recorded on a Micromass Q-TOF apparatus using electrospray ionisation (ESI) technique. Matrix assisted laser desorption ionisation time of flight (MALDI-TOF) mass spectra were recorded on a VG TofSpec spectrometer. Electrospray ionisation (ESI) was recorded on a ThermoQuest Finnigan LCQ DECA instrument. Dry column vacuum chromatography was performed on Merck Kieselgel $60(0.015-0.040 \mathrm{~mm})$, and gravity feed column chromatography was performed on Merck Kieselgel 60 (0.040-0.063 mm). Thin layer chromatography was carried out using aluminium sheets pre-coated with silica gel 60F (Merck 5554).

\section{Spectroscopy}

Absorption spectra were measured on a Perkin-Elmer Lambda $1050 \mathrm{UV} / \mathrm{Vis} / \mathrm{NIR}$ spectrometer using quartz cells with a $1 \mathrm{~cm}$ beam path. Steady state emission spectra were measured on a Horiba-Yvon FluoroLog 3 fluorescence spectrometer in $1 \mathrm{~cm}$ quartz cells in L geometry. All emission experiments were performed on solutions with an absorbance below 0.05 at the excitation wavelength. The emission quantum yields were determined using three data points from solutions of different absorbance, using 9-methylanthracene in ethanol as a reference. The emission lifetimes the anthracenes $\left(\tau^{0}\right)$ and the components of the ABA decay $\left(\tau_{\mathrm{LE}}, \tau_{\mathrm{A}}, \tau_{\mathrm{EP}}\right)$ molecules were determined using time-correlated single photon counting (TCSPC). The TCSPC traces were recorded using an add-on to the FluoroLog 3 instrument using either a $405 \mathrm{~nm}$ laser diode or a $369 \mathrm{~nm}$ diode and a PMT resulting in response times of approximately 200 ps and 1.5 ns respectively. The emission was detected through appropriate $10 \mathrm{~nm}$ bandpass filters covering the entire emission spectral range. All traces were analyzed using the PicoQuant FluoFit package version 4.1.1. The traces (typically at 5-7 different emission wavelengths) were fitted globally to a sum-of-exponentials model and evaluated by the $\chi^{2}$ value and by visual inspection of the residual function.

\section{Results and discussion}

\section{X-ray crystallography}

The ground state structures in solid phase were determined for compounds $\mathbf{4 b}, \mathbf{4 c}, \mathbf{5 b}, \mathbf{5 d}$, and 6 by single-crystal X-ray diffraction, shown in Fig. 2 and Fig. 3. [Compound 4b; CCDC 865820, compound 4c; CCDC 865819, compound 5b; CCDC 865822, compound 5d; CCDC 865821 and compound 6; CCDC 865818.] The crystal data are available as ESI (Table S1 $\dagger$ ). Single crystals suitable for X-ray diffraction were obtained from a saturated ethyl acetate solution upon slow cooling to room temperature.

It is of interest to compare the angles between the two aromatic systems within the molecule itself. In compound 6 and $\mathbf{4 b}$, an almost perpendicular disposition of the two aromatic moieties are observed with an angle of $81.3^{\circ}$ and $77.8^{\circ}$, respectively, accounting for an edge-to-face geometry between the two rings. In the case of compounds $\mathbf{5 d}$ and $\mathbf{5 b}$, this effect is not as severe and the observed angles between the anthracene and the aniline moieties are $66.6^{\circ}$ and $66.2^{\circ}$, respectively. However, in the case of compound $\mathbf{4 c}$, the aromatic ring of the aniline no longer has the same configuration of an edge-toface geometry but almost seems to adopt an edge-to-edge geometry with the two chlorine atoms pointing in opposite directions. Most likely, the ethyl group prevents the folding of the compound into the edge-to-face configuration as seen in the other structures.

A recurring motif in the crystal packing is that the anilines are edge on the anthracenes. In compound $\mathbf{5 b}$ and $\mathbf{5 d}$ two molecules pack in dimers following this motif, as seen in Fig. 3. In compound $\mathbf{4 b}$ and $\mathbf{6}$ the motif is repeated in an infinite linear chain, where the motif is repeated in a complicated interdigitated linear chain for compound 4c. The change in 


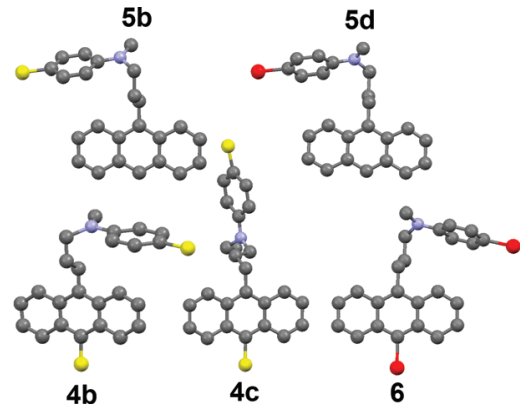

Fig. 2 Drawing of ABA molecules with $50 \%$ of probability ellipsoids. All hydrogen atoms are omitted for clarity.

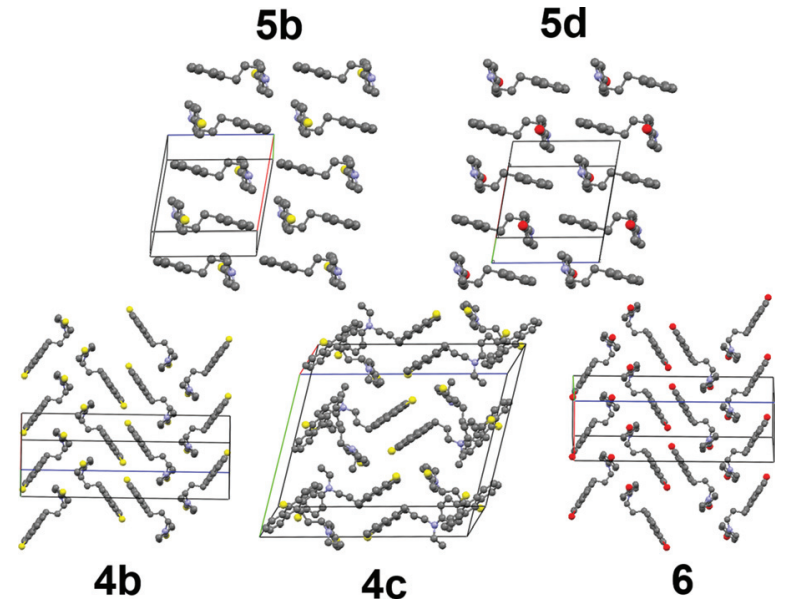

Fig. 3 Drawing of crystal packing motif in ABA molecules with $50 \%$ of probability ellipsoids. All hydrogen atoms are omitted for clarity. Red is a axis, green $b$ axis and blue $c$ axis.

packing is induced by addition of a second halogen atom when going from the dimer to the infinite chain. The complex structure is obtained by substitution of the $N$-methyl with an ethyl chain. The two more complex packing patterns also include an interchain anthracene-anthracene packing, wherein two anthracenes are fully overlapped. This is surprising as the single crystal structure of anthracene show the herringbone type packing, common to the majority of aromatic molecules.

\section{Photophysical properties of substituted anthracenes}

The three anthracene derivatives 9-methyl-anthracene (7), 9-chloro-10-methyl-anthracene (8), and 9-bromo-10-methylanthracene (9) (Fig. 4) serve as model compounds for the excited LE states of $\mathbf{5}, \mathbf{4}$, and $\mathbf{6}$, respectively. As the photophysics of anthracenes is relatively insensitive to the size of 9-alkane substituents, ${ }^{17}$ and because anthracene can be selectively excited due to the negligible low-energy spectral overlap with aniline, these compounds are assumed to be good model compounds in kinetic studies of the ABA molecules. The photophysics of anthracenes is well known, ${ }^{17}$ but for completion we will briefly summarize the main properties of the

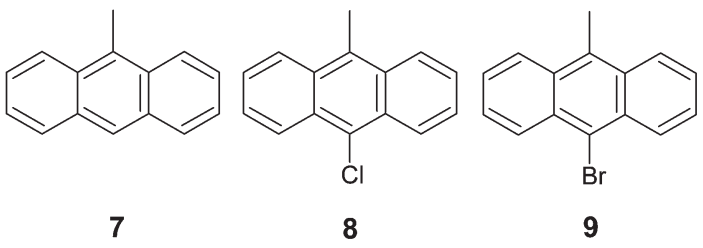

Fig. 4 Structure of the model anthracene derivatives.

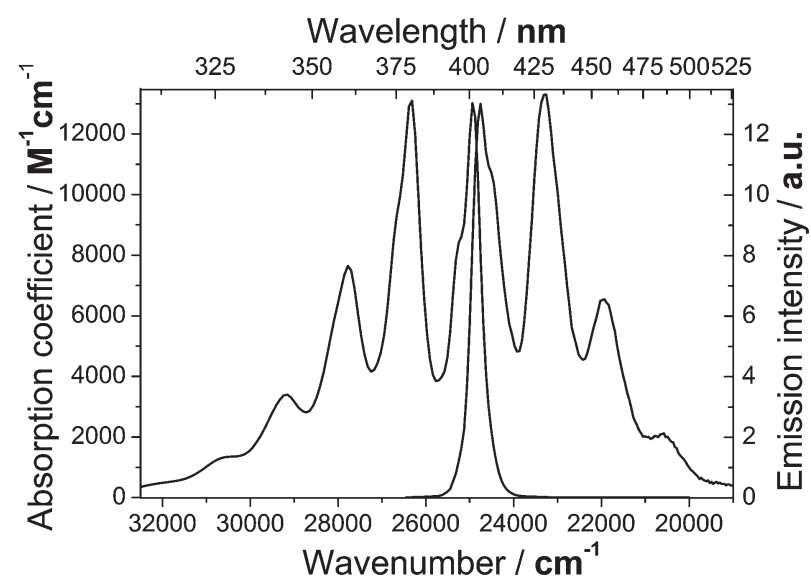

Fig. 5 Absorption (left) and emission (right) spectra of 9-chloro-10methyl-anthracene (8) in decalin.

derivatives used here. The most obvious effects of 10-halogen substitution of 9-methyl anthracene (7) is a $\sim 900 \mathrm{~cm}^{-1}$ red shift, as well as slight spectral broadening, resulting in less clearly resolved vibronic progressions. The absorption and emission spectra of compound 8, representative of the anthracenes investigated here, are shown in Fig. 5. No significant solvatochromism is observed in either absorption or emission, though a slight but noticeable red shift and broadening of the spectra is observed in toluene. This minor effect is presumably related to specific interactions between the aromatic solvent and the anthracene $\pi$-electrons. ${ }^{42}$

The excited state dynamics of the model anthracene derivatives are similarly uncomplicated. Mono-exponential fluorescence decays with approximately 5 ns lifetimes, ${ }^{16}$ depending on solvent, have been measured for compound 7 , which is in accordance with our results. Substitution of a halogen in the 10-position of compound 7 has significant consequence for the fluorescence efficiencies and excited state lifetimes, mainly through alterations to the non-radiative decay of the singlet: an approximately four-fold decrease in the non-radiative decay rate is seen on introduction of a chlorine atom at the 10-position, while a bromine substituent induces a four-fold increase in the same rate. No systematic solvent dependency on the excited state properties are introduced by halogen substitution, entirely in agreement with earlier investigations of anthracene and its derivatives. ${ }^{17}$ The complete set of photophysical properties of the anthracene derivatives investigated here are tabulated in Table 1. 
Table 1 Optical properties of substituted anthracenes in solution ${ }^{a}$

\begin{tabular}{|c|c|c|c|c|c|c|c|c|c|c|}
\hline & Solvent & $\begin{array}{l}\tilde{\nu}_{0-0}^{\mathrm{abs}} \\
\mathrm{cm}^{-1}\end{array}$ & $\begin{array}{l}\varepsilon_{0-0} \\
\mathrm{M}^{-1} \mathrm{~cm}^{-1}\end{array}$ & $f$ & $\begin{array}{l}\tilde{\nu}_{0-0}^{\mathrm{em}} \\
\mathrm{cm}^{-1}\end{array}$ & $\phi_{\mathrm{fl}}^{\mathrm{A}}$ & $\begin{array}{l}\tau^{0} \\
\mathrm{~ns}\end{array}$ & $k_{\mathrm{fl}}^{\mathrm{A}}$ & $\begin{array}{l}k_{\mathrm{fl}} / n^{2} \\
\times 10^{7} \mathrm{~s}^{-1}\end{array}$ & $\sum k_{i}$ \\
\hline \multirow{4}{*}{7} & Decalin & 25780 & 10900 & 0.12 & 25650 & 0.45 & 5.64 & 7.98 & 3.66 & 9.75 \\
\hline & THF & 25750 & 7600 & 0.09 & 25600 & 0.44 & 5.65 & 7.79 & 3.93 & 9.91 \\
\hline & meCN & 25880 & 11300 & 0.14 & 25680 & 0.34 & 5.02 & 6.77 & 3.75 & 13.15 \\
\hline & Decalin & 24930 & 13100 & 0.15 & 24760 & 0.83 & 9.53 & 8.71 & 4.00 & 1.78 \\
\hline \multirow[t]{2}{*}{8} & Toluene & 24860 & 9500 & 0.12 & 24480 & 0.71 & 11.50 & 6.17 & 2.75 & 2.52 \\
\hline & Decalin & 24890 & 11000 & 0.13 & 24540 & 0.17 & 2.03 & 8.37 & 3.84 & 40.9 \\
\hline \multirow[t]{3}{*}{9} & Toluene & 24810 & 9800 & 0.13 & 24420 & 0.23 & 2.53 & 9.09 & 4.06 & 30.4 \\
\hline & THF & 24930 & 10800 & 0.14 & 24550 & 0.18 & 2.30 & 7.83 & 3.95 & 35.6 \\
\hline & meCN & 25000 & 9400 & 0.13 & 24590 & 0.17 & 2.18 & 7.80 & 4.32 & 38.1 \\
\hline
\end{tabular}

${ }^{a}$ Transition energies, absorption coefficients, oscillator strengths, emission quantum yields, lifetimes and deactivation rates of the anthracene derivatives $\mathbf{7 ,} 8$ and $\mathbf{9}$.

\section{Photophysics of anthracene-bridge-aniline molecules}

Steady state properties. The absorption spectrum of compound 6 in decalin is shown in Fig. 6, and is typical of the spectra of all ABA molecules. A highly structured band with moderate oscillator strength appears in the absorption spectra in the $25000-30000 \mathrm{~cm}^{-1}(400-330 \mathrm{~nm})$ region, a broad low-intensity peak appears between 30000-35000 $\mathrm{cm}^{-1}$ (330-285 $\mathrm{nm})$, and a narrow and very intense peak $(\varepsilon \sim 200000$ $\left.\mathrm{M}^{-1} \mathrm{~cm}^{-1}\right)$ is at approximately $40000 \mathrm{~cm}^{-1}(250 \mathrm{~nm})$. In all solvents this spectral profile is the simple linear combination of the corresponding anthracene and aniline spectra, allowing us to assign the band at $33000 \mathrm{~cm}^{-1}(300 \mathrm{~nm})$ to the aniline $\mathrm{S}_{0}-\mathrm{S}_{1}$ transition and the structured low-energy band to the anthracene $S_{0}-S_{1}$ transition. ${ }^{47}$ The emission of compound 6 in decalin overlaid by that of compound $\mathbf{9}$ is shown in red in Fig. 6. On the basis of the considerable literature on this type of donor-acceptor systems, ${ }^{17,21,32,44}$ a broad featureless emission band from an exciplex is expected at low energies in

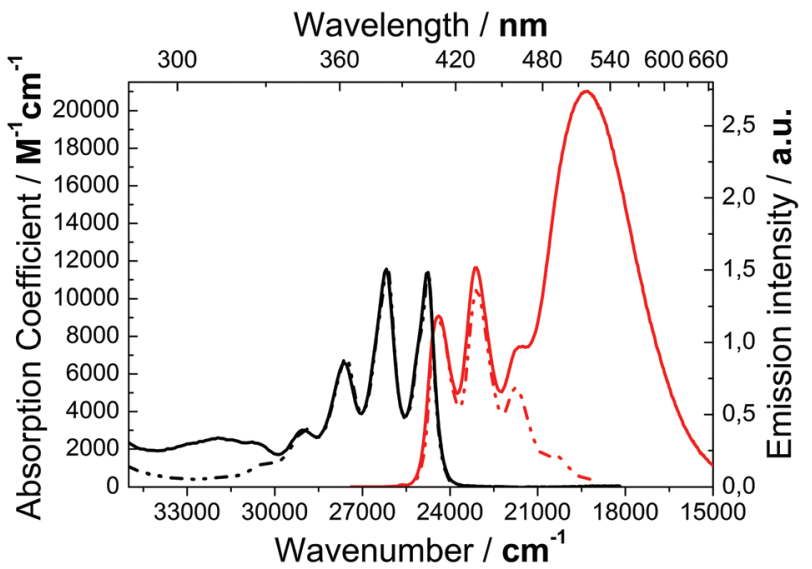

Fig. 6 Absorption (black) and emission (red) spectra of 4-bromo- $N$ - (3(10-bromoanthracen-9-yl)-propyl)- $N$-methylaniline (6) in decalin solution. Corresponding spectra of 9-methyl-10-bromo-anthracene (9) are shown as dashed lines. addition to the anthracene-like LE emission. This is the precise observation in Fig. 6, where clear deviations from mirror image symmetry are caused by an unstructured red emission band partly overlaying the anthracene emission. This low energy emission red shifts considerably as the solvent polarity increases, however the spectral shape shown in Fig. 6 is representative and similar for all ABA molecules. A summary of the steady-state absorption and emission properties of the ABA molecules are provided in Table 2.

Excited state dynamics. The excited state dynamics were investigated with time correlated single photon counting (TCSPC) measured over the entire spectral range. As LE and EP have fundamentally different emission properties, ${ }^{17,32}$ the ABA molecules exhibit complex photophysics compared to the model anthracenes, with bi- or tri-exponential TCSPC traces depending on the wavelength of observation. From global analysis of the decays, three different lifetime components could be resolved: (1) The decay of emission from the LE state $\left(\tau_{\mathrm{LE}}\right)$, (2) the grow-in of the emission of the EP state $\left(\tau_{\mathrm{A}}\right)$, and (3) the decay of the EP emission $\left(\tau_{\mathrm{EP}}\right)$. Intensity dynamics representative of the supermolecular systems in decalin are shown in Fig. 7. In decalin the short wavelength LE emission decays with lifetimes $\left(\tau_{\mathrm{LE}}\right)$ in the $\sim 2$ ns range, while the EP emission observed at longer wavelengths $\left(\tau_{\mathrm{EP}}\right)$ decays much more slowly with lifetimes in the 50-70 ns range.

For the majority of ABA molecules the TCSPC traces are similar to those of $\mathbf{5 a}$ from earlier studies, with a clear ns timescale grow-in of the EP emission intensity from zero. Close inspection of the short-time grow-in of the EP emission of 6 in decalin (Fig. 7, right) reveals however that a substantial part of the signal is formed faster than the time resolution of the system, in clear disagreement with earlier results on 5a. This extremely rapid EP formation is followed by a slower grow-in on a time-scale $\left(\tau_{\mathrm{A}}\right)$ comparable to the lifetime of the LE-state. The same two-step formation is observed for $\mathbf{4 b}$ in decalin, and is a clear indication that the excited state dynamics are more complicated than what the commonly used singlepathway structural reorganization model would imply. The life- 
Table 2 Steady state absorption and fluorescence properties of the ABA complexes ${ }^{a}$

\begin{tabular}{|c|c|c|c|c|c|c|c|c|c|}
\hline Species & Solvent & $\tilde{\nu}_{0-0}^{\mathrm{abs}}$ & $\varepsilon_{0-0}$ & $f$ & $\tilde{\nu}_{0-0}^{\mathrm{LE}}$ & $\tilde{\nu}_{\mathrm{em}}^{\mathrm{EP}}$ & $\Delta_{\mathrm{LE}-\mathrm{EP}}$ & $\Phi_{\mathrm{fl}}^{\mathrm{LE}}$ & $\Phi_{\mathrm{fl}}^{\mathrm{obs}}$ \\
\hline & Decalin & 25640 & 10300 & 0.11 & 25450 & 20370 & 5080 & 0.18 & 0.62 \\
\hline \multirow{3}{*}{$5 a$} & Toluene & 25560 & 8800 & 0.10 & 25190 & 19570 & 5620 & 0.039 & 0.42 \\
\hline & meCN & 25750 & 9200 & 0.11 & 25550 & 16400 & 9150 & 0.001 & 0.005 \\
\hline & Decalin & 25660 & 7130 & 0.09 & 25500 & 20580 & 4920 & 0.16 & 0.64 \\
\hline \multirow[t]{2}{*}{$5 b$} & Toluene & 25590 & 9950 & 0.12 & 25310 & 19940 & 5370 & 0.059 & 0.64 \\
\hline & THF & 25640 & 11860 & 0.14 & 25430 & 19190 & 6240 & - & - \\
\hline \multirow[t]{4}{*}{$5 d$} & Toluene & 25580 & 10250 & 0.12 & 25290 & 19930 & 5360 & 0.049 & 0.29 \\
\hline & THF & 25640 & 9990 & 0.12 & 25520 & 19090 & 6430 & - & - \\
\hline & meCN & 25760 & 9400 & 0.12 & 25570 & 17850 & 7720 & 0.002 & 0.010 \\
\hline & Decalin & 24840 & 11300 & 0.12 & 24390 & 19380 & 5010 & 0.23 & 0.51 \\
\hline \multirow[t]{2}{*}{$4 b$} & Toluene & 24750 & 9000 & 0.11 & 24270 & 18550 & 5720 & 0.075 & 0.32 \\
\hline & THF & 24850 & 8600 & 0.11 & 24510 & 17700 & 6810 & 0.005 & 0.11 \\
\hline & Decalin & 24750 & 11300 & 0.13 & 24390 & 19350 & 5040 & 0.14 & 0.22 \\
\hline \multirow[t]{3}{*}{6} & Toluene & 24750 & 10600 & 0.13 & 24270 & 18600 & 5670 & 0.053 & 0.14 \\
\hline & THF & 24750 & 11300 & 0.15 & 24100 & 17950 & - & 0.005 & 0.022 \\
\hline & meCN & 24850 & 10300 & 0.14 & 24570 & 17400 & 7170 & 0.002 & 0.001 \\
\hline
\end{tabular}

${ }^{a}$ All solutions flushed thoroughly with argon prior to measurement. $0-0$ transition energies, absorption coefficients, oscillator strengths, Stokes' shifts, and emission quantum yields of the LE and EP states for all investigated ABA molecules.

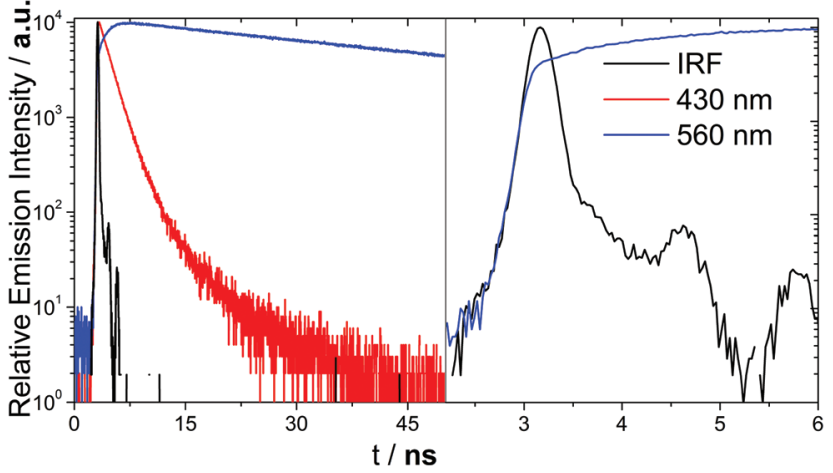

Fig. 7 Emission intensity decays of a $1 \mu \mathrm{M}$ decalin solution of 6 monitored at $430 \mathrm{~nm}$ and $560 \mathrm{~nm}$. Right panel shows detailed grow-in at $560 \mathrm{~nm}$ at short time setting.

time data are provided in Table 3, with fractional time zero amplitude of the EP emission noted in square brackets after the $\tau_{\mathrm{EP}}$-values where relevant. In THF and $\mathrm{MeCN}$ the lifetime of the LE state is too short to be measured on our instrument, but assuming that the radiative rate constant is not altered much with solvent, the emission quantum yields in these solvents imply lifetimes in the $10-50$ ps range for LE, in reasonable agreement with results from earlier transient absorption experiments. ${ }^{24,26}$

\section{Temperature and solvent polarity dependence}

The anomalous emission decay of $\mathbf{6}$ and $\mathbf{4 b}$ may potentially either be caused by the introduction of additional EP formation pathways or by significant alterations to the general
Table 3 Lifetime components of the ABA molecules

\begin{tabular}{|c|c|c|c|c|}
\hline & Solvent & $\tau_{\mathrm{LE}}$ & $\tau_{\mathrm{EP}}$ & $\tau_{\mathrm{A}}$ \\
\hline & Decalin & 1.95 & $69.3[0]$ & 1.87 \\
\hline \multirow[t]{4}{*}{$5 a$} & Toluene & 0.56 & $40.8[0]$ & 0.92 \\
\hline & THF & $-^{a}$ & 28.6 & 0.66 \\
\hline & meCN & $-^{a}$ & 5.00 & 0.17 \\
\hline & Decalin & 2.2 & $67.3[0]$ & 2.1 \\
\hline \multirow[t]{4}{*}{$5 b$} & Toluene & 0.78 & $65.8[0]$ & 0.81 \\
\hline & THF & $-^{a}$ & 18.7 & 0.89 \\
\hline & meCN & $-^{a}$ & 10.6 & 0.88 \\
\hline & Decalin & 2.6 & $64.1[0]$ & 2.4 \\
\hline \multirow[t]{4}{*}{$5 d$} & Toluene & 0.79 & $49.6[<5]$ & 0.62 \\
\hline & THF & $-^{a}$ & 16.8 & - \\
\hline & meCN & $-^{a}$ & 3.4 & 0.89 \\
\hline & Decalin & 2.3 & $75.6[15]$ & 1.9 \\
\hline \multirow[t]{4}{*}{$4 b$} & Toluene & 0.62 & $72.4[5]$ & 0.71 \\
\hline & THF & $-^{a}$ & 18.7 & 0.89 \\
\hline & meCN & $-^{a}$ & 5.15 & 0.87 \\
\hline & Decalin & 2.23 & $74.5[0]$ & 2.1 \\
\hline \multirow[t]{4}{*}{$4 c$} & Toluene & 0.68 & $67.9[0]$ & 1.12 \\
\hline & THF & $-^{a}$ & 27.4 & 1.38 \\
\hline & meCN & $-^{a}$ & 3.02 & 1.09 \\
\hline & Decalin & 1.48 & $52.0[20]$ & 1.40 \\
\hline \multirow[t]{3}{*}{6} & Toluene & 0.58 & $34.7[5]$ & 0.60 \\
\hline & $\mathrm{THF}$ & $-{ }^{a}$ & 12.1 & 0.58 \\
\hline & meCN & $-^{a}$ & 2.81 & 0.72 \\
\hline
\end{tabular}

All lifetimes in ns. The approximate amplitude (in percent of total) of the EP emission intensity at time zero is noted in parenthesis. ${ }^{a}$ Lifetime too short to be measured with our set-up.

excited state properties. While a complete analysis of this is beyond the scope of this study, we perform two simple checks to the excited state processes: the reaction barrier of the slow EP forming process is compared to reaction barriers in similar 


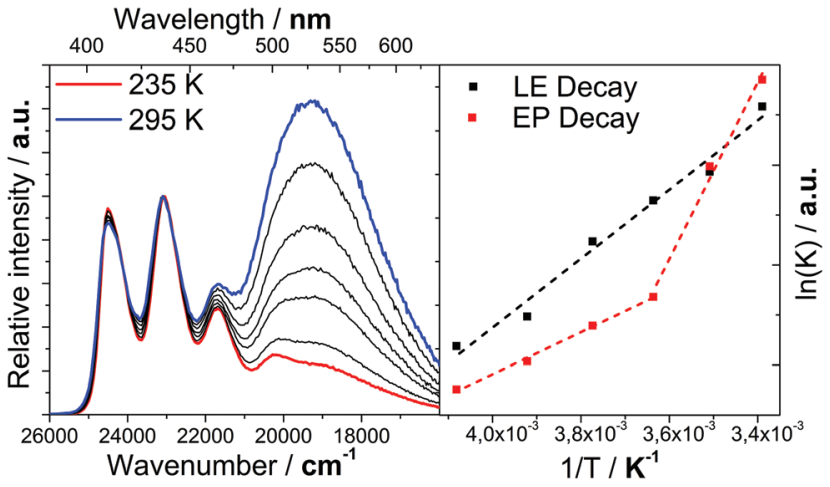

Fig. 8 Left: normalized fluorescence spectra $\left(\lambda_{\text {ex }}=405 \mathrm{~nm}\right)$ of a decalin solution of 4-bromo- $N$-(3-(10-bromoanthracen-9-yl)-propyl)$\mathrm{N}$-methylaniline (6) as a function of temperature. Right: logarithm of the total deactivation rate from the LE state (black) and the EP state (red) as function of temperature.

complexes, and we characterize the final state by its excited state dipole moment and look for significant deviations from expected values. The reaction barrier is accessible through an Arrhenius-type plot of the decay rates of LE and EP, while the excited state dipole moment is accessible from a LippertMataga plot. In accordance to the expectation from a simple internal structural reorganization process, the emission from EP decreases as the temperature is lowered (Fig. 8, left), eventually resulting in a total emission spectrum similar to the corresponding anthracene spectrum. A linear regression on the integrated emission intensity and total decay rate of the LE emission band of 6 as a function of reciprocal temperature (Fig. 8, right) yields an activation-energy for EP formation of $13.5 \mathrm{~kJ} \mathrm{~mol}^{-1}\left(\sim 1130 \mathrm{~cm}^{-1}\right)$, in good agreement with earlier studies on similar systems. ${ }^{28,48}$ The slopes of the Arrhenius plots of the rate of EP formation $\left(k_{i}=1 / \tau_{\mathrm{LE}}-1 / \tau^{0}\right)$, the total deactivation rate of $\mathrm{LE}\left(1 / \tau_{\mathrm{LE}}\right)$, and the reciprocal of the EP emission grow-in $\left(1 / \tau_{\mathrm{A}}\right)$ are similar (see ESI $\dagger$ ), implying that these processes are related, and that the "slow" EP formation is a simple process originating from LE.

The strong solvent sensitivity of the EP emission is illustrated by the representative emission spectra of compound $\mathbf{4 b}$ in Fig. 9 (left), showing a red-shift of approximately $3000 \mathrm{~cm}^{-1}$ by change of solvent from decalin to MeCN. As noted above (see Table 2), this decrease in EP emission energy with solvent polarity is accompanied by strong quenching of both LE and EP emission. This strong solvatochromism is a consequence of the charge-transfer character of the state, and the spectral shift $\left(\Delta \nu_{\mathrm{f}}\right)^{49}$ was quantitatively related to the solvent parameter $f^{\prime}\left(\varepsilon_{\mathrm{r}}, n\right)$ by eqn (2) by Lippert ${ }^{50,51}$ and Mataga. ${ }^{52,53} \varepsilon_{\mathrm{r}}$ and $n$ are here the dielectric constant and refractive index of the solvent, respectively, ${ }^{54}$ and provided that a reasonable value for the solvent cage volume $\rho^{3}$ is applied, the dipole moment of the excited state $\mu_{\mathrm{e}}$ can be obtained.

$$
f^{\prime}\left(\varepsilon_{\mathrm{r}}, n\right)=\left[\frac{\varepsilon_{\mathrm{r}}-1}{2 \varepsilon_{\mathrm{r}}+1}-\frac{n^{2}-1}{2\left(2 n^{2}+1\right)}\right]
$$

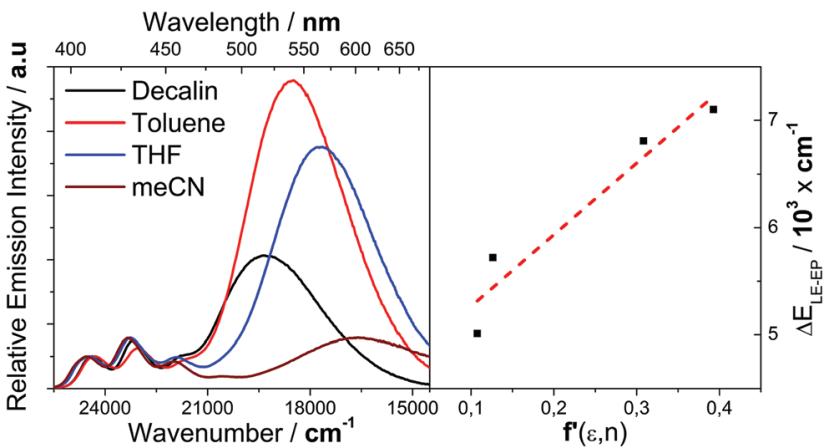

Fig. 9 Left: emission spectra of $N$-(3-(9-(10-chloroanthryl))-propyl)4-chloro- $\mathrm{N}$-methylaniline $(4 \mathrm{~b})$ in different solvents. Normalized at $410 \mathrm{~nm}$. Right: Lippert-Mataga plot of the LE-EP shift.

Table 4 Dipolar properties of exciplex states

\begin{tabular}{llrl}
\hline species & $\mu_{\mathrm{e}}^{2} / \rho^{3 a}$ & $\mu_{\mathrm{e}}{ }^{b}$ & $\Delta_{\tilde{\nu}}{ }^{c}$ \\
\hline $\mathbf{5 a}$ & 1.591 & 13.3 & 4503 \\
$\mathbf{5 b}$ & 0.914 & 10.1 & 4104 \\
$\mathbf{5 d}$ & 0.997 & 10.6 & 3933 \\
$\mathbf{4 b}$ & 0.737 & 9.1 & 4595 \\
$\mathbf{4 c}$ & 0.724 & 9.0 & 4705 \\
$\mathbf{6}$ & 0.735 & 9.1 & 4566
\end{tabular}

${ }^{a}$ Dipole squared over volume in units of $10^{-29} \mathrm{C}^{2} \mathrm{~m}^{-1}$. ${ }^{b}$ Dipole moment in Debye, calculated assuming $\rho=5 \AA$. ${ }^{c}$ Extrapolated LE-EP shift in vacuum in $\mathrm{cm}^{-1}$.

$$
\Delta \tilde{\nu}_{\mathrm{f}}=\frac{2 \mu_{\mathrm{e}}^{2}}{4 \pi \varepsilon_{0} h c \rho^{3}} f^{\prime}\left(\varepsilon_{\mathrm{r}}, n\right)
$$

The plots of $\Delta \nu_{\mathrm{f}}$ versus $f^{\prime}\left(\varepsilon_{\mathrm{r}}, n\right)$ are given in the $\mathrm{ESI} \uparrow$ and the properties extracted from the plots are compiled in Table 4, where the excited state dipole moment is extracted by assuming a constant cavity volume in all solvents. The insignificant differences in dipolar properties within each class of ABA molecules (non-, mono-, and di-halogenated) indicates that the cause of the anomalous emission decays does not affect the final emissive state.

\section{Discussion}

\section{General steady-state properties of ABA molecules}

The ABA molecules as a group show spectral properties largely analogous to that of dilute mixed solutions of aniline and anthracene. $^{55}$ No ground state association of the moieties, direct EP excitation or direct electron transfer from the ground state is apparent in the absorption spectra, showing that all light induced processes in the ABA molecules are initiated by excitation into the LE state. ${ }^{22}$ While the LE absorption maximum is red-shifted $\sim 100 \mathrm{~cm}^{-1}$ relative to the free anthracene absorption, the magnitude of this change is closer to what would be expected from a solvation- rather than a conformational effect. The presence of two overlapping emission 
bands in the ABA molecule spectra, from the anthracene-like LE on the blue side and the solvent sensitive emission of EP on the red side respectively, is in agreement with earlier studies of 5a. ${ }^{21,32,44}$ The emission yields of both LE and EP of the ABA molecules in low polarity solvents are high, implying both (1) efficient EP formation and (2) inefficient non-radiative EP deactivation. This last point is particularly notable, as the slow radiative deactivation typical of exciplexes - here illustrated by the long EP lifetimes - results in inefficient emission due to competition with oftentimes large non-radiative deactivation rates. In the present case however even double bromination of the structure induces only modest increases in non-radiative deactivation, and efficient fluorescence is observed for all species in low polarity solvents. The Arrhenius-type plots of the emission intensities and decay rates suggests a relatively straight forward interpretation of the temperature sensitive excited state processes. The LE population decays with first order kinetics in decalin, with EP formation being the dominating deactivation pathway. The plot of the deactivation rate of EP on the other hand show only weak temperature dependence below approximately $270 \mathrm{~K}$, which implies that below this temperature the thermally activated back-transfer rate $k_{-1}$ contributes insignificantly to the total EP deactivation. The activation energies extracted from these plots are in good agreement with literature values, suggesting that the thermally activated pathways are not radically altered on halogen substitution of the structures.

The strong solvent dependence of the ABA emission energy, yields, and lifetimes of both LE and EP (see Table 2 and Fig. 9) is a general phenomenon in emitter systems with donoracceptor pair character. ${ }^{19,24,26,27,30,31}$ This fact illustrates that, especially at higher solvent polarities, complete electron transfer from the aniline to the anthracene to form the zwitterion is an important deactivation pathway and must be included when analyzing the excited state processes. In this case the Lippert-Mataga plots reveals similar dipolar properties of the EP states across all ABA molecules, suggesting no qualitative changes in the product state, i.e. the anomalies in the intensity decays of $\mathbf{6}$ and $\mathbf{4 b}$ are related to alterations in the pathways from LE to EP, and not to changes in the nature of the final state.

\section{Determining rate constants for excited state processes in ABA molecules}

Even in the present favorable situation with no relevant ground state equilibria and excitation exclusively into one state, the presence of a non-negligible electron transfer state requires that a proper characterization of the excited state kinetics must include transitions between at least three excited states. After initial excitation of LE the energy dissipates through one of three channels: (1) internal deactivation via the usual radiative- or non-radiative mechanisms (IC, ISC, etc.), (2) intramolecular diffusion controlled exciplex (EP) formation, or (3) complete internal electron transfer to form the zwitterionic ET state. From the experimental data it is obvious that the rate constants connected to these processes, and thus the excited state behavior as a whole, are heavily influenced both by

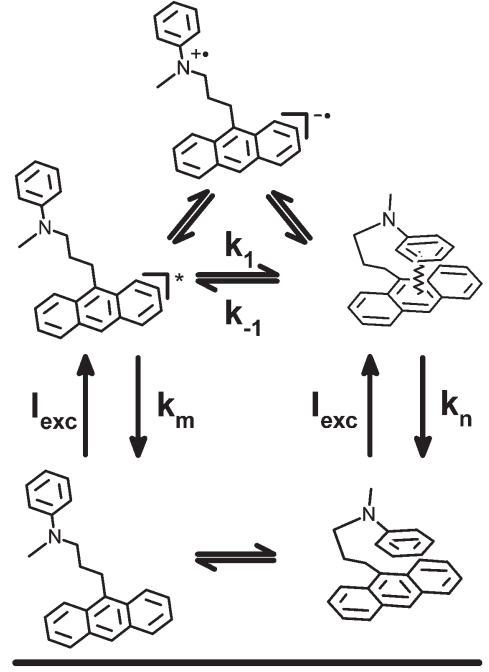

Fig. 10 The full three-excited state model. Transitions between the ground state and the charge-separated state are omitted for clarity and only rates explicitly treated in the text are labeled.

halogen substitution and the properties of the solvent. The challenge is to extract these rate constants from the experimental data, a process that in practice involves fitting a set of differential equations assumed to describe the time-evolution of the system to the TCSPC traces.

A general kinetic model assumed to be appropriate for intramolecular exciplex formation is shown schematically in Fig. 10, using ABA molecules with three excited states and a possible ground-state equilibrium as an example. The time evolution of this straight-forward model can be written as a set of three coupled differential equations. Although this set of equations has analytical solutions, the large number of parameters to fit means it is in practice difficult to extract transition rates from the TCPSC data by direct fitting without either external knowledge of some rate constants or by simplification of the model. In the case of the ABA molecules the data suggests two major simplifications for an approximate treatment: (1) The lack of absorption bands corresponding to direct excitation of EP or ET implies that the ground state equilibrium can be disregarded, and (2) at very low solvent polarities the fully charge-separated ET state is high in energy relative to $\mathrm{EP}$ and $\mathrm{LE}$, and thus energetically unavailable. These simplifications results in the two-excited-state model illustrated schematically in Fig. 11, which has been used extensively for quantitative evaluation of rate constants in photoinduced processes. ${ }^{18,32,56}$

The time-evolution of the two-excited-state model is described by the differential equations (3) and (4).

$$
\begin{gathered}
\frac{\mathrm{d}}{\mathrm{d} t}[\mathrm{LE}]=-\frac{1}{\tau_{\mathrm{LE}}}[\mathrm{LE}]+k_{-i}[\mathrm{EP}] \\
\frac{\mathrm{d}}{\mathrm{d} t}[\mathrm{EP}]=k_{i}[\mathrm{LE}]-\frac{1}{\tau_{\mathrm{EP}}}[\mathrm{EP}]
\end{gathered}
$$

Applying the boundary conditions of initial excitation of only LE and taking the emission profiles of the species into 


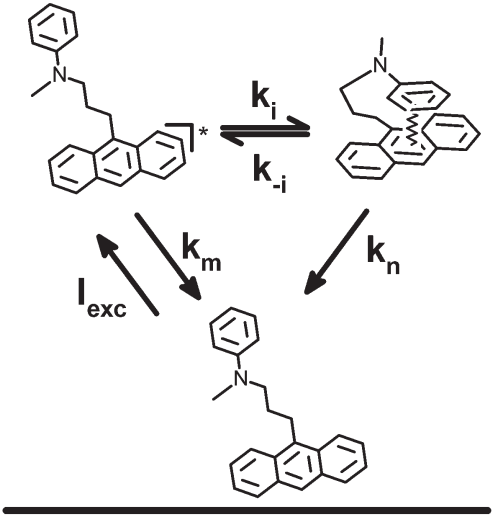

Fig. 11 The simplified two-excited state model. $k_{\mathrm{m}}$ and $k_{\mathrm{n}}$ refer to the sum of internal deactivation rates for LE and EP respectively.

account, integration of these equations leads to eqn (5) and (6) (see ESI $\uparrow$ for details), ${ }^{18,56}$ which relate the involved rate constants directly to the TCSPC traces.

$$
\begin{gathered}
I_{1}(t)=\alpha_{1}(\lambda) \mathrm{e}^{-\gamma+t}+\alpha_{2}(\lambda) \mathrm{e}^{-\gamma-t} \\
I_{2}(t)=\beta_{1}(\lambda) \mathrm{e}^{-\gamma+t}+\beta_{2}(\lambda) \mathrm{e}^{-\gamma-t} \\
\gamma_{ \pm}=\frac{1}{2}\left\{\left(\frac{1}{\tau_{\mathrm{LE}}}+\frac{1}{\tau_{\mathrm{EP}}}\right) \pm\left[\left(\frac{1}{\tau_{\mathrm{LE}}}-\frac{1}{\tau_{\mathrm{EP}}}\right)^{2}+4 k_{i} k_{-i}\right]^{1 / 2}\right\}
\end{gathered}
$$

An important feature of this model, with the current assumptions, is the prediction of an EP state that is gradually populated from zero as the aniline and excited anthracene moieties meet in diffusional encounters with rate constant $k_{i}$. Both LE and EP populations then undergo coupled, bi-exponential decays to the ground state - or dissociation to LE in the case of EP. The forward and backward rates for EP formation, $k_{i}$ and $k_{-i}$ respectively, can be extracted by fitting the expressions in eqn (5) and (6) to the fluorescence decays. The need for data fitting can further be entirely avoided in cases where the forward EP formation rate is the dominating noninternal LE deactivation rate and the internal deactivation rates are insignificantly altered relative to an appropriate model system. In this case the forward rate $k_{i}$ can be simply determined from the LE lifetime and the lifetime of the isolated model anthracene $\left(\tau_{0}\right)$ using eqn (7). ${ }^{17,57}$

$$
k_{i}=\frac{1}{\tau_{\mathrm{LE}}}-\frac{1}{\tau^{0}}
$$

In order to clearly note the difference between rates extracted by different methods, the rate constant $k_{i}$ will refer to the expression eqn (7) in the following, while the rate constants $k_{1}$ and $k_{-1}$ are reserved for the EP formation rate determined directly by fitting of differential equations to the fluorescence decays.

\section{Excited state kinetics in the ABA molecules}

We initially proceed by calculating the forward rate constant $k_{i}$ in decalin from the expression eqn (7) with the assumption of insignificant differences in the non-radiative internal deactivation of LE and model anthracenes. This simple approach provides rates in qualitative agreement with experimental observations, showing clear increases in EP emission intensity with higher forward rate constants. The two-excited-state model is however insufficient for a general description of the excited state dynamics of ABA molecules due to its inability to account for both the considerable reduction of LE (and EP) lifetimes at higher polarities and the apparent formation of a substantial fraction of the EP state of $\mathbf{6}$ and $\mathbf{4 b}$ in decalin within the lamp pulse, with no evidence of direct excitation of $\mathrm{EP}$ in the absorption spectra. Consequently, in order to extract reliable rate constants at higher solvent polarities the model used to fit the TCSPC data must contain the charge-separated ET state in addition to LE and EP. This can be accomplished by solving the differential equations describing the scheme in Fig. 10, where at higher polarities ET formation can be assumed to be irreversible (i.e. back electron transfer is to the ABA ground state) and thus contribute dark deactivation pathways for LE and EP.

The interpretation of the low-polarity TCSPC traces of $\mathbf{6}$ and 4b is less straight forward, as two processes leading to EP at very different timescales are evident: a significant fraction of the EP population is formed well within the lamp pulse (i.e. in much less than $200 \mathrm{ps}$ ), while in parallel the diffusional encounter between the excited anthracene and aniline leading to the same EP state can be observed to proceed on a ns timescale. This behavior has been observed in earlier studies, ${ }^{30-32,43,52-54}$ and is incompatible with a two-excitedstate model as shown here. The ultra-fast pathway necessary for formation of EP within the exciting pulse clearly calls for a driving force beyond random conformational diffusion. The most plausible explanation for this extremely rapid EP formation in low polarity solvents is the so-called harpooning mechanism suggested by Verhoeven et al., ${ }^{23,30,58}$ which involves complete electron transfer immediately after excitation to form the aniline radical cation and the anthracene radical anion. Driven by the large Coulombic forces involved in the ion-pair in a low polarity environment, this electron transfer state can then undergo rapid structural reorganization to form EP. In this way the formation of an ion pair constitutes a route to EP formation sufficiently rapid to compete with through-space back electron transfer. We immediately note that if a rapid EP formation pathway via ET was available to the entire ensemble of ABA molecules at all times it would dominate the LE decay, which disagrees with the observation that only part of the population follows this pathway while the remainder follows the slow process observed in all complexes. Conclusive evidence for an underlying mechanism for this division of the total population is not available in our data, however a mechanisms can be suggested by drawing analogies to the relatively considerable literature on intramolecular excimers, ${ }^{59-61}$ where it is shown that excimer formation is 
significantly influenced by the ground state configuration of the complex. If the ABA molecule ground-state configuration is defined by the specific configuration of the linker, all stable configurations can be classified by whether the two $\mathrm{C}-\mathrm{C}$ bonds in the propyl chain have trans or gauche orientation. The fully formed exciplex will then be in a gauche-gauche (gg) configuration, while the trans-trans ( $\mathrm{tt}$ ) configuration corresponds to a fully extended complex. By inspection of the ground-state structures, only in the (tg) ground-state configurations can the nitrogen lone-pair be observed close to the anthracene moiety, as $(\mathrm{gg})$ is not populated in the ground state due to steric repulsion. Thus, after photoexcitation only the ( $\mathrm{tg}$ ) population is available for the fast harpooning mechanism, while (tt) conformers must undergo at least one slow bond rotation first. As compound $\mathbf{4 c}$ would be expected to have a small (tg) population due to steric interactions with the bulkier ethyl substituent, this model further provides an explanation why significant harpooning is not observed for that compound. We note that TRXRD studies could in principle provide valuable structural evidence when evaluating such a model, as the hypothesized time-dependent changes in molecular configurations on photoexcitation would be directly observable in the scattering data.

While the above outlined model is reasonable given the literature on the subject, we note that we do not make any explicit connections between it and our kinetic model for analysis of EP formation. The transition rate constants were extracted from the TCSPC traces of compounds $\mathbf{6}$ and $\mathbf{4 b}$ decalin by applying a model where the electron transfer state ET is (partly) available and a pathway from LE to EP via ET is allowed. The differential equations describing the time evolution of this three-excited-state system were solved by a numerical method (see ESI $\dagger$ ), and the extracted (slow) rate constants are shown in Table 5. As outlined above, the EP formation rate constant calculated in this model is denoted $k_{1}$ in order to differentiate it from the corresponding rate constant $k_{i}$ calculated by eqn (7). In analogy to this the rate constant of EP dissociation into LE is denoted $k_{-1}$. We note that the backtransfer rate constant $k_{-1}$ cannot be determined with high accuracy as it is derived from the weak long-lived component

Table 5 Rate constants of all investigated complexes in decalin solution

\begin{tabular}{lcclllll}
\hline Species & $k_{i}{ }^{a}$ & $k_{\mathrm{f}}(\mathrm{LE})^{b}$ & $k_{\mathrm{f}}(\mathrm{EP})^{b}$ & $k_{1}$ & $k_{-1}$ & $k_{1} / k_{i}$ & {$[\mathrm{EP}]_{t=0}{ }^{c}$} \\
\hline $\mathbf{7}$ & - & 7.98 & - & - & - & - & - \\
$\mathbf{5 a}$ & 33.5 & 12.8 & 1.35 & 29.5 & 0.1 & 0.88 & $<5$ \\
$\mathbf{5 b}$ & 27.7 & 7.27 & 1.45 & 25.0 & 0.05 & 0.90 & 0 \\
$\mathbf{5 c}$ & 20.7 & 5.38 & 1.53 & 20.0 & 0.05 & 0.97 & 0 \\
$\mathbf{8}$ & - & 8.71 & - & - & - & - & - \\
$\mathbf{4 b}$ & 33.0 & 10.9 & 1.40 & 38.0 & 0.1 & 1.15 & $10-15$ \\
$\mathbf{4 c}$ & 34.3 & 13.0 & 1.32 & 33.0 & 0.05 & 0.96 & 0 \\
$\mathbf{9}$ & - & 8.37 & - & - & - & - & - \\
$\mathbf{6}$ & 18.3 & 9.46 & 1.80 & 7.6 & 0.2 & 0.41 & $15-20$
\end{tabular}

All rate constants in units of $10^{7} \mathrm{~s}^{-1}$. ${ }^{a}$ Two state model EP rate constant of formation: $k_{i}=1 / \tau_{\mathrm{LE}}-1 / \tau_{\mathrm{LE}}^{0} \cdot{ }^{b}$ Calculated from quantum yields and lifetimes in Tables $1-3 .{ }^{c}$ Percentage of EP at $t=0$, as estimated from intensity decays. See Fig. 10 for nomenclature. in the LE decay caused by EP dissociation followed by LE emission. Whether the values in Table 5 are reasonable was evaluated for compound $\mathbf{6}$ using variable temperature data. By assuming negligible thermal dissociation of EP to LE at low temperature, a $k_{-1}$ magnitude of $\sim 2.4 \times 10^{6} \mathrm{~s}^{-1}$ at room temperature was determined, in good agreement with the value extracted from the TCSPC traces.

The calculated EP formation rate constants in Table 5 generally show good agreement between results obtained from eqn (7) and via three-excited-state fitting. It is however clear that the EP formation rates in the three-excited state and twoexcited state models deviate considerably when there is a significant time zero EP population. We quantify this deviation by taking the ratio between the rate constants calculated with the different methods, and show the results in Table 5. The large discrepancies between $k_{1}$ and $k_{i}$, in particular for compound 6 , illustrates the importance of including a third state, and thereby the harpooning mechanism, in the model. That these very similar compounds show in some cases such different behavior suggests that energy level matching of donor aniline and acceptor anthracene is highly important in determining the excited state kinetics, and illustrates clearly that choosing a sufficiently inclusive model is crucial to extract quantitative rates in these systems.

\section{Conclusion}

In our search for suitable model systems for use in in-solution time-resolved X-ray scattering experiments we have synthesized six halogenated anthracene-bridge-aniline molecules and investigated their photophysics in solvents of different polarities. All supermolecules are upon photoexcitation capable of forming highly fluorescent exciplexes in low-polarity solvents. The temperature dependence of the EP formation revealed activation energies consistent with values for other similar complexes, and the solvent dependence of the EP emission followed the well-known expressions by Lippert and Mataga. At higher solvent polarities the formation of the ABA molecule zwitterions is favorable, and as the deactivation of the initially excited LE state become very rapid. The excited state populations of the ABA molecules thus get difficult to follow with optical spectroscopy, and as such we conclude that X-ray scattering experiments should be performed in very low polarity solvents if the scattering data is to be accurately compared to optical data. Compounds $\mathbf{5 a - d}$ and $\mathbf{4 c}$ show simple kinetic behavior in decalin, with the time-evolution after deltapulse excitation being completely characterized by a simple two-excited state model. The di-halogenated compounds $\mathbf{6}$ and 4b however exhibits surprisingly complicated photophysics even in this low-polarity solvent. The excited state behavior of these compounds was elucidated by applying the harpooningmechanism of Verhoeven, ${ }^{23,30}$ where upon photoexcitation to the LE state, a fraction of the population immediately goes to the ET state. From the ET state the population moves either to the EP by Coloumbic attraction, or by thermal relaxation to 
the ground state. As this process is extremely rapid compared to the time resolution of our instruments, it was not investigated in detail here. However, the rate constants for all processes were determined. The determination of these rate constants then allowed for modeling of the time-dependent population of all the involved states. In combination these results yielded a description of the complete low-energy excited state manifolds. The combination of the solvent response and the kinetics analyzed above demonstrated that it is possible to describe the photophysics of the ABA molecules with and without halogens in one general scheme. With the photophysics as elucidated in this work, halogen substituted anthracene-bridge-aniline compounds in non-polar solvents appear to be ideal target systems for solution phase timeresolved X-ray scattering experiments.

\section{Acknowledgements}

The authors acknowledge financial support by the Danish National Research Foundations Centre for Molecular Movies.

\section{Notes and references}

1 J. B. Birks, Excimers, Rep. Prog. Phys., 1975, 38, 903-974.

2 H. D. Becker, Unimolecular Photochemistry of Anthracenes, Chem. Rev., 1993, 93, 145-172.

3 A. Beeby, D. Parker and J. A. G. Williams, Photochemical investigations of functionalised 1,4,7,10-tetraazacyclododecane ligands incorporating naphthyl chromophores, J. Chem. Soc., Perkin Trans. 2, 1996, 1565-1579.

$4 \mathrm{~K}$. Bhattacharyya and M. Chowdhury, Environmental and Magnetic-Field Effects on Exciplex and Twisted ChargeTransfer Emission, Chem. Rev., 1993, 93, 507-535.

5 Ea. Chandros and C. J. Dempster, Intramolecular Excimer Formation and Fluorescence Quenching in Dinaphtylalkanes, J. Am. Chem. Soc., 1970, 92, 3586-3593.

6 Ea. Chandros and H. T. Thomas, Intramolecular Exciplex Formation in Naphtylalkylamines, Chem. Phys. Lett., 1971, 9, 393-396.

7 I. R. Gould, R. H. Young, R. E. Moody and S. Farid, Contact and Solvent-Separated Geminate Radical Ion-Pairs in Electron-Transfer Photochemistry, J. Phys. Chem., 1991, 95, 2068-2080.

8 F. Hirayama, Intramolecular Excimer Formation. I. Diphenyl and Triphenyl Alkanes, J. Chem. Phys., 1965, 42, 3163-3171.

9 M. H. Hui and W. R. Ware, Exciplex Photophysics 0.5. Kinetics of Fluorescence Quenching of Anthracene by n,n-Dimethylaniline in Cyclohexane, J. Am. Chem. Soc., 1976, 98, 4718-4727.

10 S. A. Jenekhe and J. A. Osaheni, Excimers and Exciplexes of Conjugated Polymers, Science, 1994, 265, 765-768.

11 D. Parker and J. A. G. Williams, Luminescence Behavior of Cadmium, Lead, Zinc, Copper, Nickel and Lanthanide
Complexes of Octadentate Macrocyclic Ligands Bearing Naphtyl Chromophores, J. Chem. Soc., Perkin Trans. 2, 1995, 1305-1314.

12 M. Sikorski, E. Krystkowiak and R. P. Steer, The kinetics of fast fluorescence quenching processes, J. Photochem. Photobiol., A, 1998, 117, 1-16.

13 M. van der Auweraer, Z. R. Grabowski and W. Rettig, Molecular-Structure and Temperature-Dependent Radiative Rates in Twisted Intramolecular Charge-Transfer and Exciplex Systems, J. Phys. Chem., 1991, 95, 2083-2092.

14 W. R. Ware, Photophysics of Exciplexes - Some Kinetic Aspects, Pure Appl. Chem., 1975, 41, 635-660.

15 A. Weller, Exciplex and Radical Pairs in Photochemical Electron-Transfer, Pure Appl. Chem., 1982, 54, 1885-1888.

16 I. B. Berlman, Handbook of Fluorescence Spectra of Aromatic Molecules, Academic Press, New York, 1965.

17 J. B. Birks, Photophysics of aromatic molecules, Wiley-Interscience, London, 1970.

18 N. Helsen, L. Viaene, M. van der Auweraer and F. C. de Schryver, Influence of the Substitution on Intramolecular Exciplex Formation Between Pyrene and Indole Moieties, J. Phys. Chem., 1994, 98, 1532-1543.

19 J. R. Larson, J. W. Petrich and N. C. C. Yang, Intramolecular Exciplexes of Polychromophoric Molecules, J. Am. Chem. Soc., 1982, 104, 5000-5002.

20 N. J. Turro, Modern Molecular Photochemistry, University Science Books Sausalito, 1991.

21 N. A. van Dantzig, H. S. Shou, J. C. Alfano, N. C. C. Yang and D. H. Levy, Photoinduced Charge-Transfer in Bichromophoric Molecules in the Gas-Phase, J. Chem. Phys., 1994, 100, 7068-7078.

22 S. L. Zhang, M. J. Lang, S. Goodman, C. Durnell, V. Fidlar, G. R. Fleming and N. C. C. Yang, Donor-acceptor interaction and photochemistry of polymethylene-linked bichromophores in solution, J. Am. Chem. Soc., 1996, 118, 9042-9051.

23 Z. R. Grabowski, Electron-Transfer and the StructuralChanges in the Excited-State, Pure Appl. Chem., 1992, 64, 1249-1255.

24 K. B. Eisenthal, Intermolecular and Intramolecular Excited State Charge Transfer, Laser Chem., 1983, 3, 145-162.

25 H. O. House, N. I. Ghali, J. L. Haack and D. Vanderveer, Reactions of the 1,8-Diphenylanthracene System, J. Org. Chem., 1980, 45, 1807-1817.

26 M. Migita, T. Okada, N. Mataga, Y. Sakata, S. Misumi, N. Nakashima and K. Yoshihara, Picosecond Laser Spectroscopy of Intramolecular Heteroexcimer Systems - TimeResolved Fluorescence Studies of Para-(Ch3)2nc6h4-(Ch2)N-(9-Anthryl), Para-(Ch3)2nc6h4-(Ch2)N-(1-Pyrenyl) Systems and 9,9'-Bianthryl, Bull. Chem. Soc. Jpn., 1981, 54, 3304-3311.

27 T. Okada, M. Migita, N. Mataga, Y. Sakata and S. Misumi, Picosecond Laser Spectroscopy of Intramolecular Heteroexcimer Systems - Time-Resolved Absorption Studies of Para-(Ch3)2nc6h4(Ch2)N(1-Pyrenyl) and Para-(Ch3)2nc6h4(Ch2)N-(9-Anthryl) Systems, J. Am. Chem. Soc., 1981, 103, 4715-4720. 
28 F. Pragst, H. J. Hamann, K. Teuchner, M. Naether, W. Becker and S. Daehne, Steady-State and Laser Spectroscopic Investigations on Intramolecular Exciplex Formation of 1-(N-P-Anisyl-N-Methyl)-Amino-3-Anthryl-(9)-Propane, Chem. Phys. Lett., 1977, 48, 36-39.

29 R. M. van der Veen, C. J. Milne, A. E. Nahhas, F. A. Lima, V.-T. Pham, J. Best, J. A. Weinstein, C. N. Borca, R. Abela, C. Bressler and M. Chergui, Structural Determination of a Photochemically Active Diplatinum Molecule by TimeResolved EXAFS Spectroscopy, Angew. Chem., Int. Ed., 2009, 48, 2711-2714.

$30 \mathrm{~J}$. W. Verhoeven, Electron-Transport Via Saturated-Hydrocarbon Bridges - Exciplex Emission from Flexible, Rigid and Semiflexible Bichromophores, Pure Appl. Chem., 1990, 62, 1585-1596.

31 J. W. Verhoeven, T. Scherer and R. J. Willemse, Solvent Effects on the Structure of Fluorescent Exciplexes in Rigidly-Bridged, Flexibly-Bridged, and Non-Bridged DonorAcceptor Systems, Pure Appl. Chem., 1993, 65, 1717-1722.

32 N. C. Yang, S. B. Neoh, T. Naito, L. K. Ng, D. A. Chernoff and D. B. Mcdonald, Chemistry of Exciplexes 0.9. Viscosity Effect on Intramolecular Exciplex Formation in SaturatedHydrocarbons, J. Am. Chem. Soc., 1980, 102, 2806-2810.

33 M. Christensen, K. Haldrup, K. Bechgaard, R. Feidenhans'l, Q. Kong, M. Cammarata, M. L. Russo, M. Wulff, N. Harrit and M. M. Nielsen, Time-Resolved X-ray Scattering of an Electronically Excited State in Solution. Structure of the 3A2u State of Tetrakis- $\mu$-pyrophosphitodiplatinate(II), J. Am. Chem. Soc., 2009, 131, 502508.

34 K. Haldrup, M. Christensen, M. Cammarata, Q. Kong, M. Wulff, S. O. Mariager, K. Bechgaard, R. Feidenhans'l, N. Harrit and M. M. Nielsen, Structural Tracking of a Bimolecular Reaction in Solution by Time-Resolved X-Ray Scattering, Angew. Chem., Int. Ed., 2009, 48, 4180-4184.

35 M. Christensen, K. Haldrup, K. S. Kjær, M. Cammarata, M. Wulff, K. Bechgaard, N. H. Harrit and M. M. Nielsen, Structure of a short-lived excited state trinuclear Ag-Pt-Pt complex in aqueous solution by time resolved X-ray scattering, Phys. Chem. Chem. Phys., 2010, 12, 6921-6923.

36 K. Haldrup, M. Christensen and M. M. Nielsen, Analysis of time-resolved X-ray scattering data from solution-state systems, Acta Crystallogr., Sect. A: Fundam. Crystallogr., 2010, A66, 261-260.

37 H. Ihee, Visualizing Solution-Phase Reaction Dynamics with Time-Resolved X-ray Liquidography, Acc. Chem. Res., 2009, 42, 356-366.

38 T. K. Kim, J. H. Lee, M. Wulff, Q. Kong and H. Ihee, Spatiotemporal Kinetics in Solution Studied by Time-Resolved X-Ray Liquidography (Solution Scattering), ChemPhysChem, 2009, 10, 1958-1980.

39 H. T. Lemke, C. Bressler, L. X. Chen, D. M. Fritz, K. J. Gaffney, A. Galler, W. Gawelda, K. Haldrup, R. W. Hartsock, H. Ihee, J. Kim, K. H. Kim, J. H. Lee, M. M. Nielsen, A. B. Stickrath, W. K. Zhang, D. L. Zhu and M. Cammarata, Femtosecond X-ray Absorption Spectro- scopy at a Hard X-ray Free Electron Laser: Application to Spin Crossover Dynamics, J. Phys. Chem. A, 2013, 117, 735-740.

40 K. Haldrup, G. Vanko, W. Gawelda, A. Galler, G. Doumy, A. M. March, E. P. Kanter, A. Bordage, A. Dohn, T. B. van Driel, K. S. Kjaer, H. T. Lemke, S. E. Canton, J. Uhlig, V. Sundstrom, L. Young, S. H. Southworth, M. M. Nielsen and C. Bressler, Guest-Host Interactions Investigated by Time-Resolved X-ray Spectroscopies and Scattering at $\mathrm{MHz}$ Rates: Solvation Dynamics and Photoinduced Spin Transition in Aqueous Fe(bipy)(3)(2+), J. Phys. Chem. A, 2012, 116, 9878-9887.

41 M. Vanderauweraer, L. Viaene, P. Vanhaver and F. C. Deschryver, Influence of Solvent Polarity on the Radiationless Decay of the Intramolecular Exciplexes of OmegaPhenyl-Alpha-N,N-Dimethylaminoalkanes, J. Phys. Chem., 1993, 97, 7178-7184.

42 J. Hinatu, H. Masuhara, N. Mataga, Y. Sakata and S. Misumi, Absorption-Spectra of Intermolecular and Intramolecular Exciplex Systems of Pyrene and N,N-Dimethylaniline in Alcoholic Solutions, Bull. Chem. Soc. Jpn., 1978, 51, 1032-1036.

43 N. C. C. Yang, D. W. Minsek, D. G. Johnson, J. R. Larson, J. W. Petrich, R. Gerald and M. R. Wasielewski, Photochemistry of Polychromophoric Arylamines, Tetrahedron, 1989, 45, 4669-4681.

44 F. Pragst, H. J. Hamann, K. Teuchner and S. Daehne, Structural Effects on Intra-Molecular Exciplexes of 1-Amino3-Anthryl-(9)-Propanes, J. Lumin., 1978, 17, 425-437.

45 A. C. Spivey, A. J. Bissell and B. Stammen, A practical synthesis of N-p-anisyl-N-methyl-amino-3-(9-anthryl)-propane: An exciplex fluorescence 'thermometer', Synth. Commun., 1998, 28, 623-632.

46 K. Gen, S. Mitsuharu, T. Naoto, F. Yasushi, N. Tadao, I. Tatsuro, N. Yukinori and T. Akio, New Fluorine-Containing Anthracene Comound and Method for manufacturing the same, Kanto Denka Kogyo Co Ltd, Taiho Ind co Ltd, Japan, 2002.

47 J. R. Platt, Classification of Spectra of Cata-Condensed Hydrocarbons, J. Chem. Phys., 1949, 17, 484-495.

48 B. Wegewijs, R. M. Hermant, J. W. Verhoeven, A. G. M. Kunst and R. P. H. Rettschnick, Determination of the Barrier to Intramolecular Exciplex Formation in a JetCooled, Bichromophoric Molecule, Chem. Phys. Lett., 1987, 140, 587-590.

49 It is convenient in complex systems such as the anthracene-bridge-aniline molecules to report the energy shift of the EP state relative to the $\mathrm{S} 1(0-0)$ transition of the LE state. The model is derived under the assumption of a vanishing ground state dipole moment of the component responsible for the LE emission. When the shift is reported relative to the LE state as opposed to the EP emission in vacuum, it is also a requirement that there is no emission solvatochromy of that state. Both of these requirements are fulfilled for anthracene. This relatively simple model should therefore suffice to semi-quantitatively describe the 
electronic changes in the complexes in different polarity environments.

50 E. Lippert, Dipolmoment Und Elektronenstruktur Von Angeregten Molekulen, Z. Naturforsch., A: Astrophys. Phys. Phys. Chem., 1955, 10, 541-545.

51 E. Lippert, Habilitationsschrift zur Erlangung der Lehrberichtigung (Venia Legendi) fur das Fach Physikalische Chemie Ander Techischen-Hochschule-Stuttgart - Spektroskopische Bestimmung des Dipolmomentes Aromatischer Verbindungen im Ersten Angeregten Singulettzustand, Z. Elektrochem., 1957, 61, 962-975.

52 N. Mataga, Y. Kaifu and M. Koizumi, The Solvent Effect on Fluorescence Spectrum - Change of Solute-Solvent Interaction during the Lifetime of Excited Solute Molecule, Bull. Chem. Soc. Jpn., 1955, 28, 690-691.

53 N. Mataga, Y. Kaifu and M. Koizumi, Solvent Effects Upon Fluorescence Spectra and the Dipolemoments of Excited Molecules, Bull. Chem. Soc. Jpn., 1956, 29, 465-470.

54 N. Mataga, H. Chosrowjan and S. Taniguchi, Ultrafast charge transfer in excited electronic states and investigations into fundamental problems of exciplex chemistry: Our early studies and recent developments, J. Photochem. Photobiol., C, 2005, 6, 37-79.

55 J. B. Birks, Photophysics of aromatic molecules, Wiley-Interscience, London, New York, 1970.
56 W. R. Laws and L. Brand, Analysis of 2-State Excited-State Reactions - Fluorescence Decay of 2-Naphthol, J. Phys. Chem., 1979, 83, 795-802.

57 J. R. Lakowicz, Principles of Fluorescence Spectroscopy, Springer-Verlag New York Inc., New York 3, revised edn, 2006.

58 B. Wegewijs, R. M. Hermant, J. W. Verhoeven, M. P. Dehaas and J. M. Warman, Exciplex-Type Emission from Folded and Extended Conformations of a Donor-Acceptor Molecule with Limited Flexibility, Chem. Phys. Lett., 1990, 168, 185-190.

59 M. Vanderauweraer, A. Gilbert and F. C. Deschryver, Influence of Starting Conformations on Intramolecular Exciplex Formation in Omega-Phenyl-Alpha-N,N-Dimethylaminoalkanes, J. Am. Chem. Soc., 1980, 102, 4007-4017.

60 F. C. Deschryver, L. Moens, M. Vanderauweraer, N. Boens, L. Monnerie and L. Bokobza, Kinetic and Thermodynamic Aspects of Excimer Formation in 2,4Diphenylpentanes as Polystyrene Models, Macromolecules, 1982, 15, 64-66.

61 F. C. Deschryver, P. Collart, J. Vandendriessche, R. Goedeweeck, A. M. Swinnen and M. Vanderauweraer, Intramolecular Excimer Formation in Bichromophoric Molecules Linked by a Short Flexible Chain, Acc. Chem. Res., 1987, 20, 159-166. 